\title{
Algebraic spinors on Clifford manifolds
}

\author{
J S R Chisholm \\ Institute of Mathematics and Statistics, University of Kent, Canterbury, Kent, CT2 7NF, UK
}

\begin{abstract}
A Clifford manifold of $n$ dimensions is defined by the fundamental relation $\left\{\mathbf{e}_{\mu}(x), \mathbf{e}_{\sqrt{ }}(x)\right\}=2 \mathrm{~g}_{\mu r}(x) 1$ between the $n$ frame field components $\left\{e_{\mu}(x)\right\}$ and the metric matrix $\left\{g_{\mu v}(x)\right\}$. At any point $x$, the tangent space, orthonormal frames, and the spin group are defined in terms of the frame field. Different types of field are classified in terms of their properties under the general linear coordinate transformation group on the manifold, and under spin group transformations.

Connections for different types of field are determined by their covariance properties under these two groups. The bivector spin connection is then uniquely determined by the 'Uniformity Assumption' for Clifford algebraic grades. A key result is established, that the frame field is necessarily covariantly constant on a Clifford manifold, with both vector and spin connections.

'Spin elements' are formed by contracting the frame field with Riemannian vector fields, and possess a 'two-sided' commutator covariant derivative. A set of Riemannian fields orthonormal with respect to the manifold defines an orthonormal set of spin elements in the tangent space, from which idempotents can be constructed. If $\mathbf{S}$ is an asymptotically flat $\mathrm{n}-1$ dimensional submanifold on which a constant idempotent is defined in terms of a constant spin frame, parallel transport along geodesics from each point of $S$ defines a unique position-dependent extension of the idempotent in a patch $\mathrm{P}$ of the manifold.

In an earlier model which describes the electroweak interactions of leptons, with a simplification of the Glashow Lagrangian, the 'right-hand' part of the two-sided spin connection gives rise to new gravitational terms. The nature of these new terms is discussed.
\end{abstract}

Keywords: Manifold, frame field, Clifford algebra, algebraic spinors, parallel transport, spin group, idempotents, cosmological constant, electroweak interactions

MSC numbers: 11E88, 15A63, 15A66, 53B15, 53B25, 53Z05, 53C07, 53C60, 53C80, 83E15

\section{Clifford Manifolds}

In a series of papers, the author, in collaboration with Dr Ruth Farwell, has developed models of the elementary particles of one family and their interactions [1-6]. Our aims have been:

(a) to base each model on a single specific Clifford algebra, using the unique irreducible representation, with fermions described by the Dirac equation;

(b) to derive the observed interactions, including gravitation, from gauge groups generated within the chosen algebra;

(c) to avoid the introduction of unobserved particles such as supersymmetric particles, or complex structures such as strings.

The inclusion of gravitation led us to develop a simple structure which we have called a Clifford manifold, and later we gave an informal description of this concept [7] While it is possible to formulate this idea within the standard fibre bundle context, Clifford manifolds then appear to be degenerate, as we shall see. 
Several standard concepts enter the description of Clifford manifolds. An n-dimensional manifold consists of a union of a countable number of patches, and each patch corresponds to a continuous range of $n$ real variables $x \equiv\left\{x^{\mu} ; \mu=0,1,2, \ldots, n-1\right\}$. In this paper we consider only a single patch $P$, and do not study global manifold properties.

The fundamental equation defining a Clifford manifold is the anticommutation relation

$$
\left\{\mathbf{e}_{p}(x), \mathbf{e}_{v}(x)\right\}=21 \mathrm{~g}_{\mu v}(x) \text {, }
$$

relating the non-degenerate metric matrix $g_{\mu v}(x), \mu, v=0,1,2, \ldots, n-1$, and the 'frame field' elements $e_{\mu}(x)$. At each point $x$, the frame field elements are assumed to span a linear space, the tangent space at $x$. Their vector transformation properties under non-degenerate coordinate transformations are detailed below. The elements $e_{\mu}(x)$, for fixed $x$, are also assumed to multiply associatively, and so to generate a Clifford algebra, as described in Section 2. Throughout this paper, bold symbols are used to indicate elements of the local algebra. The symbol 1 represents the universal unit of the algebra, and is common to all points $\mathrm{x}$; the consistency of this assumption will be discussed in Section 8. The specific inclusion of this universal element is important in the discussion of the transformation properties of different types of geometro-physical fields. As we shall detail below and in Section 2 , the elements $\mathrm{e}_{\mu}(\mathrm{x})$ transform non-trivially not only as vectors under the group of coordinate transformations, but also as vectors under spin group transformations in the tangent space. We shall refer to vectors under the coordinate transformation group as 'Riemannian vectors', but, because of the spin group property, we also say that $\mathrm{e}_{\mu}(\mathrm{x})$ are 'spin vectors'. The double classification of fields under coordinate and spin group transformations is of fundamental importance.

The other basic equation defines the increment of displacement on $\mathbf{P}$ as

$$
\mathrm{d} s=\mathbf{e}_{\mu}(\mathrm{x}) \mathrm{dx} \mathbf{x}^{\mu}
$$

If we consider (1.1) in the standard differential geometrical context, the metric matrix would enter the definition of a metrical base space. However, we shall see that the frame field automatically defines the spin structure, which is normally defined separately as a bundle. Since, in our models using higher-dimensional spaces, gauge groups also arise from the frame field, it is impossible to separate the fibre structure from the base space. All geometro-physical entities arise from the two relations (1.1) and (1.2).

The patch $P$ can be covered by a new real coordinate system $\left\{y^{y}(x) ; v=0,1,2, \ldots, n-1\right\}$ provided that the partial derivatives $\partial y^{v} / \partial x^{\mu}$ are continuously differentiable a sufficient number of times, and the Jacobian determinant $\left|\partial y^{\nu} / \partial x^{\mu}\right|$ is non-zero. Then the increments of the two coordinate systems are related by

$$
d y^{\nu}=\left(\partial y^{v} / \partial x^{\mu}\right) d x^{\mu}
$$

We note that $\left\{d x^{\mu}\right\}$ and the coordinate derivatives $\left\{\partial_{\mu}\right\}$ are not, as in some formulations of differential geometry, taken to be Grassmannian vectors or covectors. All anti-commutation properties stem from the basic relations (1.1).

The coordinate transformations (1.3) belong to the group GL(n), and the increment of length ds defined by (1.2) is postulated to be invariant under this group. It follows that the frame field vectors $\mathbf{e}_{\mu}(x)$ transform contragrediently under coordinate transformations:

$$
\hat{\mathbf{e}}_{v}(\mathrm{y})=\left(\partial x^{\mathrm{H}} / \partial \mathrm{y}^{\mathrm{v}}\right) \mathbf{e}_{\mu}(\mathrm{x}) \text {. }
$$

In space-time, ds is the increment of proper time, and is, correctly, invariant under coordinate transformations. This group of transformations is fundamental to one of the basic principles governing physical theories:

PRINCIPLE 1: Any equation describing a physical system must transform covariantly under the general linear group of coordinate transformations 
This principle is very familiar, and is, for example, basic to General Relativity.

Since the metric is non-degenerate on $P$, it has a definite fixed signature $(p, q)$, with $p+q=n$, and this signature is unchanged under real coordinate transformations, since the basic relation (1.1) ensures that $\left(\mathrm{g}_{\mu \mathrm{v}}\right)$ transforms as a tensor.

\section{Tangent Space and the Spin Group}

At any particular point $x$ of $P$, the frame field components

$$
\mathbf{e}_{\mu}(\mathrm{x})(\mu=0,1,2, \ldots, \mathrm{n}-1)
$$

span the $n$-dimensional tangent space $T_{x}$ at $x$. We can therefore define real linear combinations of the set $\left\{e_{\mu}(x)\right\}$ which form orthonormal sets of vectors $\left\{c_{r}(x) ; r=0,1,2, \ldots, n-1\right\}$ in $T_{x}$, satisfying the orthonormality relations

$$
\left\{c_{r}(x), c_{s}(x)\right\}=21 \eta_{r s}
$$

where $\eta_{r s}$ is the Minkowski metric matrix $\operatorname{Diag}(1, \ldots, 1,-1, \ldots,-1)$, with signature $(p, q)$. The algebra generated by the spin vectors $\mathrm{c}_{\mathrm{r}}(\mathrm{x})$ is, for each $\mathrm{x}$, the Clifford Algebra $\mathrm{Cl}_{\mathrm{p}, \mathrm{q}}$.

The linear relations between the two sets of basis vectors can be expressed as

$$
\mathbf{e}_{\boldsymbol{p}}(\mathrm{x})=\mathrm{h}_{\mu}{ }^{\mathrm{T}}(\mathbf{x}) \mathbf{c}_{\mathbf{r}}(\mathrm{x}) \text {, }
$$

where the coefficients $h_{\mu}{ }^{r}(x)$ constitute the 'vielbein field'. If we substitute (2.3) into (1.1) and use (2.2), we obtain the standard relation

$$
\eta_{\mathrm{rs}} \mathrm{h}_{\mu}{ }^{\mathrm{r}}(\mathrm{x}) \mathrm{h}_{\mathrm{v}}{ }^{\mathrm{s}}(\mathrm{x})=\mathrm{g}_{\mu \mathrm{v}}(\mathrm{x})
$$

The set of orthonormal frames $\left\{c_{r}(x)\right\}$ satisfying (2.2) have both physical and mathematical significance The continuous group of transformations between different orthonormal frames is generated by the bivectors $c_{\mathrm{rs}}(x) \equiv c_{r}(x) c_{s}(x)(r<s)$ of the algebra, and is the spin group $\operatorname{Spin}(p, q)$, a subgroup of the Clifford-Lipschitz group [8]. If $\Lambda(x)$ is an element of the group, an arbitrary spin vector $c(x)=\Sigma a^{r} c_{r}(x)$ transforms to

$$
\check{\mathbf{c}}(\mathrm{x})=\boldsymbol{\Lambda}(\mathrm{x}) \mathbf{c}(\mathrm{x}) \boldsymbol{\Lambda}^{-1}(\mathrm{x}) \text {. }
$$

We note that an orthonormal set of spin vectors, satisfying (2.2), remains orthonormal under this group. A 'two-sided' spin group transformation (2.5) is equivalent to a 'one-sided' operation of a matrix which acts as a rotation on the column formed from the components $c_{r}(x)$ of $c(x)$. This justifies calling $\mathbf{c}(\mathbf{x})$ a 'spin vector'.

We define the operation of the spin group on $\mathbf{e}_{\mu}(\mathrm{x})$ as the operation (2.5) on each basis vector $\mathbf{c}_{\mathrm{r}}(\mathrm{x})$, the coefficients $h_{\mu}{ }^{r}(x)$ being kept fixed. It would be possible to transform the coefficients $h_{\mu}{ }^{r}(x)$ by a matrix operation on the superfix ' $r$ ', cancelling the rotational operation on the components $c_{r}(x)$. Fixing $h_{\mu}{ }^{r}(x)$ makes precise the 'spin vector' property of $e_{\mu}(x)$. We note that the algebraic unit 1 is invariant under a spin group transformation of the form (2.5).

Physically, the algebra $\mathrm{Cl}_{1,3}$ is the algebra corresponding to the space-time of Special Relativity, and the group Spin $(1,3)$ is the proper Lorentz group of space rotations and boosts, relating different orthonormal frames $\left\{\mathbf{c}_{r}\right\}$. This group also plays an important role in General Relativity, as Weinberg [9] , building on ideas of Utiyama [10] and Kibble [11], has emphasised: the set of space-time coordinate frames in which there is, locally, zero gravitational force, are related by the Lorentz group, of which $\operatorname{Spin}(1,3)$ is the continuous part.

More generally, An orthonormal basis $\left\{\boldsymbol{c}_{\mathbf{r}}\right\}$ satisfying (2.2) delineates the grades of an algebra, since the basis elements of grade $k$ are just products of $k$ different basis vectors. Products of $k$ vectors of a non-orthonormal basis $\left\{\mathbf{e}_{\mu}\right\}$ are in general of mixed grade.

This physical and mathematical significance of orthonormal bases underlies the second principle which we postulate to be fundamental to physical theories: 
PRINCIPLE 2: Any equation describing a physical system must be covariant under transformations of the relevant spin group $\operatorname{Spin}(p, q)$.

Weinberg [9] has noted the independence of Principles 1 and 2, and that, in space-time, each is basic to theories involving gravitation and particles with spin. Earlier, Kibble [11] considered covariance under the Lorentz group operating on column states, together with local coordinate transformations under Poincaré algebra; he did not introduce the concept of a curved manifold initially, but the Poincare transformations were eventually interpreted as 'general coordinate transformations', and the Greek suffixes involved were associated with a space-time dependent metric. Kibble also noted the independent action of the coordinate and spin groups, in agreement with Weinberg. For Kibble, however, the vierbein field appears as the gauge field of the translation part of the Poincare group; this contrasts with the appearance of the vielbein field as coefficients in our relations (2.3) between the frame field and the orthonormal frames characteristic of the Clifford structure. We shall discuss this difference in Section 10.

In order to demonstrate the independence of the coordinate and spin groups, we list in the Table below several classes of algebraic quantity, together with their behaviour under transformations of the coordinate and spin groups. As in the main text of the paper, Greek suffixes label vector and tensor components; their transformation properties under the coordinate group are based on (1.4). Covariant and contravariant vector fields, with components $V_{v}(x)$ and $W^{\lambda}(x)$ respectively, can be introduced, as for Riemannian manifolds, through the increment of distance $V_{v}(x) d x^{y}$ and directional derivative $W^{\lambda}(x) \partial / \partial x^{\lambda}$. The components $W^{\lambda}$ and $V_{v}$ have, as usual, coordinate group transformation rules similar to (1.3) and (1.4). On a Clifford manifold, 'Riemannian vectors' are defined to contain the factor 1 , ensuring that they have trivial scalar transformation properties under spin group transformations. In contrast, the spin group action on $\mathbf{e}_{\mu}(x)$ is of the 'two-sided' form (2.5); this action distinguishes the frame field from Riemannian fields. As for Riemannian vectors, the metric matrix in the basic equation (1.1) also occurs with a factor 1, indicating that it is also a spin scalar.

In our earlier models $[1,2,3,4]$, column spinors represented fermions. The one-sided action of the spin group on column spinors, and on conjugate row spinors, is discussed in Section 3.

\begin{tabular}{|l|l|l|l|}
\hline Element & Name & Coordinate group & Spin group \\
\hline $\mathbf{g}_{\mu v}(\mathbf{x}) \mathbf{I}$ & Metric tensor & Symmetric tensor & Scalar \\
\hline $\mathrm{V}_{\mu}(\mathbf{x}) \mathbf{I}$ & Riemannian vector & Covariant vector & Scalar \\
\hline $\mathbf{e}_{\mu}(\mathbf{x})$ & Frame field vector & Covariant vector & Two-sided \\
\hline$\psi(\mathbf{x})$ & Column spinor & Scalar & One-sided \\
\hline
\end{tabular}

In Sections 3 and 4, we shall link 'connections' on a Clifford manifold to transformation properties under the two groups (coordinate and spin). In Sections 6 and 7, we shall see that the algebraic spinors we define have two-sided spin group transformations of the form (2.5), and in consequence have two-sided spin connection, different to the 'one-sided' spin connection of column spinors.

\section{Connections: the Uniformity Assumption}

It is well known that, on a Riemannian manifold, the coordinate derivatives $\partial_{\mu} \equiv \partial / \partial x^{\mu}$ are not covariant, and have to be replaced by covariant derivatives if Principle 1 is to be satisfied. The covariant derivatives of covariant and contravariant fields, with components $V_{\mu}(x)$ and $W^{\lambda}(x)$ respectively, on a Riemannian manifold are defined in terms of the Riemannian connection 
They are

$$
\Gamma_{\mu v}^{\lambda}=1 / 2 g^{\lambda \sigma}\left(\partial_{\mu} g_{v \sigma}+\partial_{v} g_{\mu \sigma}-\partial_{\sigma} g_{\mu v}\right)
$$

and

$$
\mathrm{D}_{\mu} \mathrm{V}_{v}=\partial_{\mu} \mathrm{V}_{\mathrm{v}}-\Gamma_{\mu v}{ }^{\lambda} \mathrm{V}_{\lambda}
$$

On a Clifford manifold, these fields are multiplied by the spin scalar 1 , which is invariant under spin transformations and also under displacements on the manifold, leaving these covariant derivatives essentially unchanged. So if (3.2) and (3.3) are multiplied by 1, they then apply to Clifford manifolds.

In the Table above, it was noted that the spin group action on a column spinor is one-sided, compared with the two-sided action (2.5) on an element of the algebra. Specifically, the one-sided action on a column spinor $\psi(\mathrm{x})$ and a bar-conjugate (generalised Dirac conjugate) row spinor is

$$
\psi(x) \rightarrow \Lambda(x) \psi(x), \bar{\varphi}(x) \rightarrow \bar{\varphi}(x) \Lambda^{-1}(x)
$$

In space-time, when the spin group action is interpreted as a Lorentz transformation, this one-sided action accords with the experimental properties of electrons and other fermions.

It has usually been assumed that gauge transformations of spinors are also of the form (3.4), since a right-handed action on a column spinor is restricted to scalar multiplication. However, we have recently studied the possibility of using algebraic spinors, defined as left ideals of an algebra, instead of column spinors [12]. Idempotents, and hence ideals of the algebra, are themselves elements of the algebra, in contrast to column spinors, which are acted on by elements of the algebra. In quantum mechanical language, algebraic spinor 'states' belong to the same algebra as the 'operators'. For this reason, we examined the postulate that algebraic spinors undergo two-sided gauge transformations of the form (2.5). These two-sided gauge transformations, applied [12] to our model of Glashow's electroweak interactions [13], led to a simplification of the gauge generator. The effect of using two-sided gauge transformations will be discussed more fully in Section 9. For now, we simply note that a change to two-sided gauge transformations necessarily gives rise to a change in the form of the spinor connection.

To derive the covariant derivative of a column spinor $\psi$ which is subject to a one-sided gauge transformation (3.4), we shall follow the spirit of Weinberg's argument [14], which depends upon Principles 1 and 2. Weinberg, however, does not assume the properties of a Clifford manifold. He bases his work on the 'tetrad field', consisting of four Riemannian vector fields, which do not have the same spin transformation properties as the frame field. He then introduces spin separately. This procedure accords with the fibre bundle concepts of separated base space (tetrad field) and fibre (spin). As we have already emphasised, Clifford manifolds, based upon the frame field, incorporate all properties in the definitions (1.1) and (1.2).

Weinberg derived a unique covariant derivative using Principles 1 and 2 . In the context of this paper, however, the derivation of this unique result depends on making a further assumption about the derivatives of physical fields:

\section{UNIFORMITY ASSUMPTION: The derivative $\partial_{\mu} \mathbf{F}$ of any field $\mathbf{F}$ of grade $\mathrm{k}$ in a Clifford algebra is also of grade $\mathrm{k}$.}

This assumption ensures that a displacement of a field on the manifold does not change the grade of the field. For example, the electromagnetic field, which is a bivector field in space-time, is a bivector field everywhere and for all time. Geometrically, the non-preservation of grades would mean that physical dimensionality changed from point to point. We are accustomed to accepting 
the analogous property when we consider Riemannian vector fields, which are assumed to possess the vector property everywhere. In the absence of the Uniformity Assumption, algebraic grades could be mixed through automorphisms of the algebra. If, as we assume, the physical dimensionality of an algebraic quantity is related to the algebraic grade, grade mixing would lead to puzzling geometro-physical consequences, as noted by Pezzaglia [15]. In his exploration of invariance under the automorphic group of the algebra, he generalises our notion of a 'Clifford manifold'. In the present paper, we maintain the original definition, so that Clifford algebraic vectors are distinguished by their appearance in (1.1) over a patch.

If a column spinor undergoes a one-sided gauge transformation (3.4), it is necessary to introduce a gauge potential with components $\mathbf{G}_{\mu}(\mathbf{x})$ in order to define a covariant derivative

$$
\delta_{\mu} \psi \equiv \partial_{\mu} \psi+\mathbf{G}_{\mu}(\mathbf{x}) \psi
$$

Then, when $\psi$ undergoes the gauge transformation, $\mathbf{G}_{\mu}(\mathbf{x})$ must transform according to the usual rule,

$$
\mathbf{G}_{\mu} \rightarrow \mathbf{\Lambda} \mathbf{G}_{\mu} \mathbf{\Lambda}^{-1}-\left(\partial_{\mu} \mathbf{\Lambda}\right) \mathbf{\Lambda}^{-1}
$$

A transformation $\Lambda(x)$ is generated by bivectors $c_{r s}$ and an infinitesmal transformation is of the form

Then the infinitesmal form of $(3.6)$ is

$$
\Lambda(x)=1+1 / 2 \omega^{\mathrm{rs}} \mathbf{c}_{\mathrm{rs}} \equiv \mathbf{1}+\omega(\mathrm{x})
$$

$$
\mathbf{G}_{\mu} \rightarrow \mathbf{G}_{\mu}+\left[\boldsymbol{\omega}, \mathbf{G}_{\mu}\right]-\partial_{\mu} \boldsymbol{\omega} .
$$

In addition to satisfying the transformation rule (3.8), $\mathbf{G}_{\mu}$ must be a coordinate vector. Further, since, by the Uniformity Assumption, $\partial_{\mu} \omega$ is coordinate vector and a Clifford (or spin) bivector, $\mathbf{G}_{\mu}$ must be a coordinate vector which is the sum of a spin bivector and an arbitrary spin scalar field. This arbitrary vector field has sometimes been identified in space-time, by other authors, as the electromagnetic potential, but in this paper it is assumed to be identically zero.

To identify the bivector field $\mathbf{G}_{\mu}$, note that $\mathbf{e}^{v}\left(\partial_{\mu} \mathbf{e}_{\boldsymbol{\nu}}\right)$ is a bivector plus a scalar, and that it transforms by

$$
\mathbf{e}^{\mathrm{v}}\left(\partial_{\mu} \mathbf{e}_{\mathrm{v}}\right) \rightarrow \mathbf{e}^{\mathrm{v}}\left(\partial_{\mu} \mathbf{e}_{\mathrm{v}}\right)+\left[\omega, \mathbf{e}^{\mathrm{v}}\left(\partial_{\mu} \mathbf{e}_{\mathrm{v}}\right)\right]+\mathbf{e}^{\mathrm{v}}\left[\partial_{\mu} \omega, \mathbf{e}_{\mathrm{v}}\right]
$$

Since $\partial_{\mu} \omega$ is a bivector, the generalisation (A1) of old formulae [16,17], established in Appendix $A$ of this paper, gives, with $n=0$ and $n=2$,

Thus (3.9) becomes

$$
\mathbf{e}^{\mathrm{v}}\left[\partial_{\mu} \omega, \mathbf{e}_{v}\right]=\mathbf{e}^{\mathrm{v}} \partial_{\mu} \omega \mathbf{e}_{v}-\mathrm{n} \partial_{\mu} \omega=-4 \partial_{\mu} \omega \text {. }
$$

Likewise

$$
\mathbf{e}^{\mathrm{v}}\left(\partial_{\mu} \mathbf{e}_{v}\right) \rightarrow \mathbf{e}^{\mathrm{v}}\left(\partial_{\mu} \mathbf{e}_{v}\right)+\left[\omega, \mathbf{e}^{\mathrm{v}}\left(\partial_{\mu} \mathbf{e}_{v}\right)\right]-4 \partial_{\mu} \omega \text {. }
$$

$$
\left(\partial_{\mu} \mathbf{e}_{v}\right) \mathbf{e}^{v} \rightarrow\left(\partial_{\mu} \mathbf{e}_{v}\right) \mathbf{e}^{v}+\left[\omega,\left(\partial_{\mu} \mathbf{e}_{v}\right) \mathbf{e}^{v}\right]+4 \partial_{\mu} \omega .
$$

Forming the bivector commutator from these expressions, we see that the expression

$$
1 / 8\left[\mathbf{e}^{v},\left(\partial_{\mu} \mathbf{e}_{v}\right)\right]
$$

gives the correct transformation (3.8), and is the only bivector containing the derivative $\partial_{\mu}$ which does so. However (3.11) is not covariant under coordinate transformations. So the unique bivector satisfying the two covariance conditions and the Uniformity Assumption, is

$$
\mathbf{G}_{\mu}=1 / 8\left[\mathbf{e}^{v},\left(D_{\mu} \mathbf{e}_{v}\right)\right] \text {, }
$$

where $D_{\mu}$ is the Riemannian covariant derivative defined by (3.2).

The expression (3.12) is well known, but Fairchild [18] has given a more general form for the spin connection, showing that the Uniformity Assumption needs to be made in order to establish the uniqueness of (3.12). 


\section{The Frame Field Connection: the Covariant Constancy Identity}

In space-time, frame field components $\mathbf{e}_{\mu}$ are represented by the Dirac matrices $\boldsymbol{\gamma}_{\mu}$ on the manifold, and the connection for these matrices has been studied by many authors. Shortly after Dirac's theory of the electron was published, Fock and Ivanenko [19] discussed the covariant derivative of spinors, introducing the matrices $\gamma_{\mu}$ both as generalisations of the Dirac matrices and as vectors defining the element of length of form (1.2) in the 'Geometrie quantique Lineare' of space-time. At that time, it was not generally realised that the Dirac algebra was a particular Clifford algebra, but Fock and Ivanenko were in fact treating space-time as a Clifford manifold. The phrase 'quantum geometry' also foreshadows the more general concept of non-commutative geometry developed by Connes [20] and others, which offers an approach to the global properties of manifolds. When Clifford algebras are introduced within this more general framework, the resulting structure appears to be similar to a Clifford manifold.

The geometric interpretation of $\gamma_{\mu}$, or more generally $\mathbf{e}_{\mu}$, has been emphasised by, for example, H.S. Green [21] in discussing teleparallelism, by Hestenes and Sobczyk [22], and as the 'frame field' in our own work [2,7]. Chern [23] has used the 'frame field' as a basic geometrical concept, but for him, the component vectors are not assumed to satisfy the basic Clifford relation (1.1).

In 1932, Schrodinger [24] discussed the covariant derivative of $\gamma_{\mu}$ in space-time, and showed that it had to be of the form

$$
\nabla_{\mu} \gamma_{v}=\partial_{\mu} \gamma_{v}-\Gamma_{\mu v}^{\lambda} \gamma_{\lambda}+\left[\mathbf{M}_{\mu}, \gamma_{v}\right]
$$

where $\mathbf{M}_{\mu}$ is a 'vector of matrices'. In addition, he stated that it was necessary for this equation to satisfy Principles 1 and 2 . The generalisation of (4.1) to a manifold of arbitrary dimensionality is

$$
\nabla_{\mu} e_{v}=\partial_{\mu} e_{v}-\Gamma_{\mu \nu}^{\lambda} e_{\lambda}+\left[M_{\mu}, e_{v}\right]
$$

and we can derive the value of $\mathbf{M}_{\mu}$ from the fact that the product $\mathbf{e}_{v} \psi$ is assumed to exist and to be a column spinor. Since it is also a coordinate vector, its covariant derivative, containing both Riemannian and spin connections, is

$$
\partial_{\mu}\left(\mathbf{e}_{\nu} \psi\right)-\Gamma_{\mu \nu}{ }^{\lambda}\left(\mathbf{e}_{\lambda} \psi\right)+\mathbf{G}_{\mu}\left(\mathbf{e}_{\nu} \psi\right)
$$

where $\mathbf{G}_{\mu}$ is given by (3.12). The three forms of covariant derivative (3.5), (4.2) and (4.3) are mutually consistent only if $\mathbf{M}_{\mu}=\mathbf{G}_{\mu}$, so that (4.2) becomes

$$
\nabla_{\mu} \mathbf{e}_{v}=\partial_{\mu} \mathbf{e}_{v}-\Gamma_{\mu v} \mathbf{\lambda}_{\lambda}+\left[\mathbf{G}_{\mu}, \mathbf{e}_{v}\right] \text {, }
$$

Not surprisingly, the spin connection for a frame field vector $\mathbf{e}_{\mu}$ is the same as that for a column spinor $\psi$. However, while $\mathbf{G}_{\mu}$ acts only on the left on $\psi$ in (3.5), it has a 'two-sided' action in the commutator in (4.4). This accords with the two-sided action of the spin group operator in (2.5): the commutator form in (4.4), together with the Riemannian connection, ensures that $\nabla_{\mu} \mathbf{e}_{\nu}$ is covariant under both coordinate and spin groups. Note that the vector connection $\Gamma_{\mu \nu}{ }^{2}$ is the standard Riemannian connection (3.1), symmetrical in the lower suffixes; it is well known that the spin connection term $\left[\mathbf{G}_{\mu}, \mathbf{e}_{v}\right]$ in (4.4) can be related to the introduction of torsion, but it is treated separately in this paper.

We have derived the form of $\mathbf{G}_{\mu}$ by assuming the two covariance principles and the Uniformity Assumption. An alternative approach, followed by Brill and Wheeler [25], Fletcher [26], Loos [27] and ourselves [2], is to assume that the frame field is parallel transported, so that $\nabla_{\mu} e_{\nu}=0$, or from (4.4) and (3.2),

$$
\mathrm{D}_{\mu} \mathbf{e}_{v}+\left[\mathbf{G}_{\mu}, \mathbf{e}_{\mathrm{v}}\right]=0 .
$$

Forming the contracted commutator of this equation with $\mathbf{e}^{\mathrm{v}}$ gives

$$
\left[\mathbf{e}^{\mathbf{v}}, D_{\mu} \mathbf{e}_{v}\right]+\left[\mathbf{e}^{\mathbf{v}},\left[\mathbf{G}_{\mu}, \mathbf{e}_{v}\right]\right]=0 \text {. }
$$


If we now make the Uniformity Assumption, it follows that $\mathbf{G}_{\mu}$ is a bivector (apart from the arbitrary Riemannian vector field), and, again using (Al) with $n=0$ and $n=2$, this equation reduces to

so that $\mathbf{G}_{\mu}$ is again given by $(3.12)$.

$$
\left[\mathbf{e}^{v}, D_{\mu} e_{v}\right]-8 G_{\mu}=0 \text {, }
$$

However, the fact that (3.12) can be derived from (4.5) by contraction does not ensure that (3.12) satisfies (4.5), which has to be true for all values of $\mu$ and $v$. On a Clifford manifold, (4.5) is in fact an identity. However, the proof is non-trivial, so we state the result as a formal theorem, giving the proof in Appendix $B$.

\section{Theorem 1: The Covariant Constancy Identity}

The bivector spin connection

$$
\mathbf{G}_{\mu}=1 / 8\left[\mathbf{e}^{\mathrm{v}},\left(\mathbf{D}_{\mu} \mathbf{e}_{v}\right)\right] \text {, }
$$

given by (3.12), identically satisfies (4.5) on a Clifford manifold, so that

$$
\nabla_{\mu} \mathbf{e}_{\nu} \equiv \partial_{\mu} \mathbf{e}_{v}-\Gamma_{\mu v}{ }^{\lambda} \mathbf{e}_{\lambda}+\left[\mathbf{G}_{\mu}, \mathbf{e}_{v}\right] \equiv 0
$$

It is essential to note that the proof of this theorem depends upon (1.1), and so is only true for Clifford manifolds, or when some equivalent assumption is made. Since the derivation of (3.12) is based on the symmetry and uniformity principles, these principles and equation (1.1) establish (4.6) is an identity satisfied by a previously determined function $\mathrm{G}_{\mu}$. It is important to emphasise the contrast with the common approach in which (4.5) is assumed, with (3.12) as a consequence.

Geometrically, (4.6) ensures that the frame field $\left\{\mathbf{e}_{\mathrm{v}}\right\}$ is necessarily covariantly constant on a Clifford manifold. At first sight, this may seem a surprising result. But, in essence, we have defined the manifold to be the frame field, so that (4.6) can be seen as a consistency condition - the frame field is covariantly constant relative to itself.

Differentiating (1.1) and using (1.1) itself, we find that the identity (4.6) implies the standard assumption of covariant constancy of the metric tensor:

$$
D_{\mu} \mathbf{g}_{\mu \mathrm{v}} \equiv 0 \text {. }
$$

However, (4.6) is a result involving both the metric and the spin structure, and so is a stronger condition than (4.7).

The Covariant Constancy Identity is central to the results of later sections of this paper, in which we define algebraic spinors on a Clifford manifold in terms of Riemannian vector fields. Riemannian vectors have only the Riemannian connection, and spinors have only a spin connection. We shall use (4.6), which incorporates both connections, to link vector fields with algebraic spinor fields.

\section{Orthonormal Vector Fields}

In this section, we summarise some known results about Riemannian vector fields and geodesics. For the present, we omit the Clifford scalar 1 from our definition of Riemannian fields.

Corresponding to a non-null incremental displacement $\mathrm{dx}^{\mu}$ a manifold, the increment of "proper time' $\mathrm{dt}$ is defined by

$$
\mathrm{dt}^{2}= \pm \mathrm{g}_{\mu \nu} \mathrm{dx}^{\mu} \mathrm{dx} \mathrm{x}^{\nu}
$$

with plus (minus) sign corresponding to a time (space)-like displacement. The corresponding velocity components 
satisfy

$$
\dot{x}^{\mu}=\mathrm{dx} / \mathrm{dt}
$$

$$
\mathrm{g}_{\boldsymbol{\mu} v} \dot{\boldsymbol{x}}^{\mu} \dot{\mathrm{x}}^{\nu}= \pm 1
$$

Now suppose that a smooth curve $\mathrm{C}$ has $\dot{x}^{\mu}$ tangent at each point $\mathrm{x}$. Then a vector $\mathrm{W}^{v}$ is parallel transported along $\mathrm{C}$ if it satisfies

$$
\dot{x}^{\mu} D_{\mu} W^{\nu}(x) \equiv \partial W^{\nu}(x) / \partial t+\dot{x}^{\mu} \Gamma_{\mu}{ }^{\nu}(x) W^{\lambda}(x)=0
$$

at every point of $C$. If the velocity $\dot{x}^{\mu}$ is itself parallel transported, so that (5.4) becomes

$$
\ddot{x}^{\nu}+\Gamma_{\mu \nu}{ }^{\nu}(\mathrm{x}) \dot{x}^{\mu} \dot{x}^{\lambda}=0,
$$

then $\mathrm{C}$ is a geodesic, and $\mathrm{t}$ is a corresponding affine parameter.

At any non-degenerate point on an $\mathbf{n}$-dimensional manifold, $\mathbf{n}$ non-null incremental displacements $\mathrm{dx}_{\mathrm{r}}{ }^{\mu}(\mathrm{r}=0,1, \ldots, \mathrm{n}-1)$ can be chosen which are mutually orthogonal relative to the manifold metric. Then the corresponding velocity components

$$
\mathrm{W}_{\mathrm{r}}^{\mu}=\dot{x}_{r}^{\mu} \quad(\mathrm{r}=0,1, \ldots, \mathrm{n}-1)
$$

satisfy the orthonormality relations

$$
\mathrm{g}_{\mu v} \mathrm{~W}_{\mathrm{r}}^{\mu} \mathrm{W}_{\mathrm{s}}^{\mathrm{v}} \equiv \mathrm{g}_{\mu \nu} \dot{x}_{r}^{\mu} \dot{x}_{s}^{\nu}=\eta_{\mathrm{rs}}
$$

where $\eta_{\mathrm{rs}}$ is the Minkowski matrix corresponding to the signature $(p, q)$.

If the $\mathrm{n}$ vectors satisfying (5.7) are parallel transported along a curve $\mathrm{C}$ with tangent $\mathrm{W}_{0}{ }^{\mu} \equiv \dot{x}_{0}{ }^{\mu}$, then they will satisfy (5.4); The curve $\mathrm{C}$ will be the geodesic (5.5) with $\dot{x}^{\mu} \equiv \dot{x}_{0}{ }^{\mu}$, and the corresponding 'proper time' $t$ will be the affine parameter.

We note two important properties:

(a) From (5.4), it follows that the inner products of Riemannian vectors, and hence the orthonormality relations (5.7), are preserved under parallel transport.

(b) Since any incremental displacement initially orthogonal to the geodesic tangent $\mathrm{W}_{0}{ }^{\mu}$ has components of the form

$$
\delta x^{\mu}=\sum_{r=1}^{n-1} \dot{x}_{r}^{\mu} \delta_{r}=\sum_{r=1}^{n-1} W_{r}^{\mu} \delta_{r}
$$

for some increments $\delta_{\mathrm{r}}$, the displacement is orthogonal to the geodesic everywhere on $\mathrm{C}$ :

$$
\mathrm{g}_{\mu v} \mathrm{~W}_{\mathrm{o}}{ }^{\mu} \delta \mathrm{x}^{\nu}=0 .
$$

In physical applications, we frequently wish to know how a function given on a space-like surface can be extended to space-time. More generally, Wald [28] has discussed a method of extending a function given on a smooth non-degenerate 'surface' $S$, part of an n-1 dimensional submanifold, to a tubular region of an $n$ dimensional manifold $M$. It is assumed that $S$ is parametrised by the $n-1$ coordinates $x^{\mu}(\mu=1,2, \ldots, n-1)$, and that the remaining continuous coordinate on $M$ is taken to be $x^{0}=t$, an affine parameter corresponding to geodesic displacements, starting in a normal direction from each $x$ on the surface $S$. Then, given a smooth field $W^{\mu}\left(t=0, x_{1}, x_{2}, \ldots, x_{n-1}\right)$ on $S$, it can be smoothly extended by parallel transport to some tubular neighbourhood $0<t<t_{0}$ of $S$, on which the geodesic structure is non-degenerate $[28,29]$. This ensures the existence of an $\mathrm{n}$-dimensional patch $\mathrm{P}$ on which the parallel transported field $\mathrm{W}^{\mu}\left(t, \mathrm{x}_{1}, \mathrm{x}_{2}, \ldots, \mathrm{x}_{\mathrm{n}-1}\right)$ is a well-defined smooth function of the $n$ parameters.

We wish to use this method to extend $\mathrm{n}$ fields of the form (5.6), satisfying the orthonormality relations (5.7) on $S$. Then property (a) above will ensure that (5.7) is satisfied on the patch $P$. Since the metric is assumed non-degenerate, it is always possible to choose $n$ fields (5.6) which satisfy 
(5.7) at every point $x$ of $S$. For example, the fields can be chosen at one particular point $x_{0}$ on $S$, and extended smoothly by parallel transport to a neighbourhood of $x_{0}$ [30]. However, for a general manifold, there is no simple unambiguous 'recipe' for relating the fields (5.6) at different points of $\mathrm{S}$. As we shall see in the next section, this ambiguity is also a problem when we come to define an equivalent on a manifold of a 'constant idempotent', on which the concept of an algebraic spinor depends.

This ambiguity does not arise if the manifold $M$ is flat in some $n-1$ dimensional region. If the surface $S$ lies in this flat region, the fields (5.6) can be parallel transported, by translation, in every direction throughout $\mathbf{S}$, defining a 'constant' Riemannian frame there. It is then possible to use geodesic parallel transport from the points of $S$, as described above, to define a unique frame throughout a patch $P$, which is an extension of the constant frame on $S$. Property (b) above ensures that the orthonormality (5.9) of incremental displacemants on $S$ to the geodesics also applies to the parallel transported displacements. It can also be shown $[28,29]$ that the points on the geodesics with the same value of the affine parameter $t$ form a smooth surface $S_{t}$ which is orthonormal to the geodesics. So the orthogonality properties of the geodesics, the fields $W_{r}^{\mu}$, and the surface $S$, are true of every surface $S_{t}$ in the patch $P$.

\section{Spin Elements}

One important fact about the identity (4.6) is that the spin connection $\mathbf{G}_{\mu}$ appears in the commutator, in contrast to the 'one-sided' occurrence in (3.5). In this section, we shall define 'spin elements', which also have a two-sided spin connection of commutator form. Then in Section 7 , these spin elements will be used to define idempotents, and hence algebraic spinors $\psi$ on a Clifford manifold, which again have a two-sided commutator spin connection of form

$$
\left[\mathbf{G}_{\mu}, \boldsymbol{\Psi}\right] \text {. }
$$

We use bold type for an algebraic spinor since, unlike a column spinor, it is a true element of the algebra. In (6.1), in addition to the usual bivector interaction term $G_{\mu} \Psi$ for a spinor, an extra 'righthanded' interaction term $-\psi \mathbf{G}_{\mu}$ appears. The effects of the extra interaction term will be discussed in Section 9. For the present, we simply note that the 'two-sided' interaction term (6.1) is of the same form as a general form of interaction introduced recently [12] in flat spaces, on a quite different basis. We argued that 'two-sided' gauge potentials for algebraic spinors, of the form

$$
\left[\mathbf{A}_{\mu}, \boldsymbol{\Psi}\right]
$$

were consistent with the fact that these spinors $\boldsymbol{\psi}$ were constructed from general elements of a Clifford algebra, which have two-sided gauge transformations. So, if we interpret (6.1) as a gravitational interaction of the spinor $\Psi$, it is consistent with the form (6.2) already proposed for flat spaces.

A 'spin element' is formed by contracting the frame field with a contravariant Riemannian vector field with components $\mathrm{W}^{\prime}(\mathrm{x})$, giving

$$
\mathbf{W}(x)=\mathbf{W}^{v}(x) \mathbf{e}_{v}(x)
$$

which is a coordinate scalar and a spin vector. This contraction is similar to that used by Bade and Jehle [31] in deriving 'second rank spinors' from 'world vectors' in their discussion of quadratic forms of vector fields.

Using the definition (6.3) and the Covariant Constancy Identity (4.6),

$$
\partial_{\mu} \mathbf{W}(\mathbf{x})+\left[\mathbf{G}_{\mu}(\mathbf{x}), \mathbf{W}(\mathbf{x})\right]=\left\{\partial_{\mu} \mathbf{W}^{\nu}(\mathbf{x})\right\} \mathbf{e}_{\nu}(\mathbf{x})+\mathbf{W}^{\nu}(\mathbf{x})\left\{\partial_{\mu} \mathbf{e}_{\nu}(\mathbf{x})+\left[\mathbf{G}_{\mu}(\mathbf{x}), \mathbf{e}_{\nu}(\mathbf{x})\right]\right\}
$$




$$
=\left\{\partial_{\mu} W^{\nu}(x)+\Gamma_{\mu \rho}{ }^{\nu} W^{\rho}(x)\right\} e_{n}(x) .
$$

This contains the covariant derivative of $W^{\prime}(x)$, and is therefore doubly covariant, being both a coordinate vector and a spin vector, but the spin connection on the left of (6.4) is of two-sided commutator form. We shall be considering products of a set of spin elements $\left\{\mathbf{W}_{\mathrm{r}}(\mathbf{x}) ; \mathrm{r}=0,1,2, \ldots\right\}$ of the form (6.3), formed from Riemannian vector fields with components $W_{r}^{\prime}(x)$. When we differentiate a product of spin elements using Leibnitz' rule, the derivative $\partial_{\mu}$ can be considered as an operator commuting through the product, and each separate derivative $\left(\partial_{\mu} \mathbf{W}_{x}\right)$ can be appropriately expressed as a commutator $\left[\partial_{\mu}, W_{r}\right]$. Then the covariant derivative of $W_{r}(x)$, on the left of (6.4), is written as the commutator

$$
\left[\partial_{\mu}+\mathbf{G}_{\mu}(\mathbf{x}), \mathbf{W}_{\mathrm{r}}(\mathbf{x})\right]
$$

So the operator $\partial_{\mu}+\mathbf{G}_{\mu}$ acts as a derivation, and we can use Leibnitz' rule to form the covariant derivative

$$
\left[\partial_{\mu}+\mathbf{G}_{\mu}, \prod_{r} \mathbf{W}_{\mathrm{r}}(\boldsymbol{x})\right]
$$

of a product of several spin elements. The expression (6.6) is covariant, since each term in the Leibnitz expansion contains a single covariant derivative of form (6.5). We have therefore established:

\section{Theorem 2}

If $\left\{\mathbf{W}_{\mathrm{r}}(\mathrm{x}) ; \mathrm{r}=0,1,2, \ldots\right\}$ are a set of spin elements corresponding to Riemannian vector fields with components $\mathrm{W}_{\mathrm{r}}(\mathrm{x})$, then the covariant derivative of any polynomial $\mathrm{p}\left\{\mathrm{W}_{\mathrm{r}}(\mathrm{x})\right\}$ in the corresponding spin elements is

with two-sided spin connection.

$$
\left[\boldsymbol{\delta}_{\mu}, \mathrm{p}\left\{\mathbf{W}_{\mathbf{r}}(\mathbf{x})\right\}\right] \equiv\left[\partial_{\mu}+\mathbf{G}_{\mu}(\mathbf{x}), \mathrm{p}\left\{\mathbf{W}_{\mathrm{r}}(\mathbf{x})\right\}\right],
$$

To establish a further key theorem, consider the Clifford scalar product of two spin elements. From the definition (5.3) and the fundamental equation (1.1),

$$
\begin{gathered}
\mathbf{W}_{\mathrm{r}}(\mathbf{x}) \cdot \mathbf{W}_{\mathrm{s}}(\mathbf{x})=1 / 2\left\{\mathbf{W}_{\mathrm{r}}(\mathbf{x}), \mathbf{W}_{\mathrm{s}}(\mathbf{x})\right\} \\
=1 / 2 \mathbf{W}_{r}^{\mu}(\mathbf{x}) \mathbf{W}_{\mathrm{s}}^{v}(\mathbf{x})\left\{\mathbf{e}_{\mathrm{H}}(\mathbf{x}), \mathbf{e}_{\mathrm{v}}(\mathbf{x})\right\} \\
=\mathbf{g}_{\mu v} \mathbf{W}_{r}^{\mu}(\mathbf{x}) \mathbf{W}_{\mathrm{s}}^{v}(\mathbf{x}) \mathbf{1} .
\end{gathered}
$$

So we have established:

\section{Theorem 3}

If $\mathrm{W}_{\mathrm{r}}^{\mu}(\mathrm{x})$ are the components of a set $(r=0,1,2,$. .) of Riemannian vector fields, the Clifford scalar product $\mathbf{W}_{\mathrm{r}}(\mathrm{x}) \mathbf{W}_{\mathrm{s}}(\mathrm{x})$ of any two of the corresponding spin elements has magnitude equal to the inner product of the Riemannian fields relative to the manifold metric.

In Section 5, we introduced sets of $\mathbf{n}$ Riemannian fields satisfying the orthonormality relations (5.7). Theorem 3 ensures that the corresponding spin elements satisfy the tangent space orthonormality relations, similar to (2.2):

$$
\mathbf{W}_{\mathrm{r}}(\mathrm{x}) \cdot \mathrm{W}_{\mathrm{s}}(\mathrm{x})=\eta_{\mathrm{rs}} 1 \text {. }
$$

Further, if the Riemannian fields are chosen to satisfy (5.7) on an n-1 dimensional surface $S$, and are geodesically parallel transported, as in Section 5 , to a surface $S_{t}$, then, using (6.4), it follows that the spin elements $\mathrm{Wr}(\mathrm{x})$ are parallel transported, satisfying the equation

$$
\dot{x}^{\mu}\left[\partial_{\mu}+\mathbf{G}_{\mu}(\mathbf{x}), \mathbf{W}_{\mathrm{r}}(\mathbf{x})\right]=0 .
$$


Using (3.12), this equation can be expressed in terms of the frame field and its derivatives:

$$
\partial \mathbf{W}_{\mathrm{r}} / \partial \mathrm{t}+1 / 8 \dot{x}^{\mu}\left[\left[\mathbf{e}^{\mathrm{v}},\left(\mathrm{D}_{\mathrm{\mu}} \mathbf{e}_{\mathrm{v}}\right)\right], \mathbf{W}_{\mathrm{r}}\right]=0 \text {. }
$$

Likewise, any polynomial in the set $\left\{W_{r}\right\}$ is parallel transported to $S_{t}$ by

$$
\dot{x}^{\mu}\left[\partial_{\mu}+\mathbf{G}_{\mu}(\mathbf{x}), \mathrm{p}\left\{\mathbf{W}_{\mathrm{r}}(\mathbf{x})\right\}\right]=0 .
$$

The constancy of the scalar products $W_{\mathrm{r}}(\mathrm{x}) . \mathrm{W}_{\mathrm{s}}(\mathrm{x})$ in (6.9) under parallel transport can be checked directly, using (6.10).

\section{Definition of Idempotents and Algebraic Spinors}

In a flat $n$-dimensional space, an algebraic spinor $\psi(x)$ is of the form

$$
\Psi(x)=\mathbf{E}\left(x, c_{r}\right) \mathbf{U}\left(c_{r}\right)
$$

where $\mathbf{E}\left(\mathbf{x}, \mathbf{c}_{\mathrm{r}}\right)$ is a general position-dependent element of the appropriate Clifford algebra, and $\mathbf{U}\left(\mathbf{c}_{\mathbf{r}}\right)$ is a constant idempotent satisfying

$$
\mathbf{U}^{2}=\mathbf{U}
$$

The spinor is defined in terms of a chosen orthonormal vector basis, satisfying (2.2), which is constant in the flat space.

When we are dealing with a curved Clifford manifold, the fact that each point of the manifold has a separate tangent space is crucially important. In flat spaces, the algebras in the tangent spaces can be uniquely related by translation to define a 'constant algebra'. For regions of general curved manifolds, there is no unique way of relating the algebras in the different tangent spaces. The problem is analogous to defining a manifold generalisation of a plane wave, which possesses translational invariance in flat spaces. This analogy prompts the suggestion that an idempotent, and hence an algebraic spinor, be defined on a non-degenerate flat surface $S$, part of an n-1 dimensional flat submanifold. We therefore assume that the patch $P$ contains such a surface $S$ Then the definitions of idempotents and spinors can be uniquely extended over the patch by the method described in Sections 5 and 6.

This procedure is similar to that used in the definition of an S-matrix, where interactions are assumed to be negligible for asymptotic 'incoming' and 'outgoing' states. In this paper, we make no attempt at a rigorous analytic study of asymptotic conditions. However, we note that the problems of self-interaction and long-range forces will arise, as in S-matrix theory. It is also important to remember that the Bondi-Metzner-Sachs asymptotic group structure is by no means simple [32].

We assume that, on $\mathbf{S}$, a constant idempotent $\mathbf{U}\left(\mathbf{c}_{\mathbf{r}}\right)$ is defined in terms of translation-invariant vector basis $\left\{\boldsymbol{c}_{\mathrm{r}}\right\}$. This vector basis is then identified on $S$ with a set of spin elements

$$
\mathbf{W}_{r}=\mathbf{c}_{r} \quad(r=0,1, \ldots . n-1) \text {, }
$$

which satisfy the correct orthonormality relations (6.9). From the definition (6.3) of spin elements, it follows that the associated Riemannian fields are given generally by

$$
W_{r}^{\mu}(x) 1=e^{\mu}(x) \cdot W_{r},
$$

so that on S, or 'asymptotically',

$$
W_{r}^{\mu}(x) 1=e^{k}(x) \cdot c_{r}
$$

where

$$
e^{\mu}(x)=g^{\mu v}(x) e_{v}(x)
$$

Through (7.3) and (7.5), the asymptotic spin elements and fields are given in terms of the chosen asymptotic spin vector basis and the frame field on $\mathrm{S}$. But these spin elements and fields are parallel transported geodesically by $(6.11)$ and $(5.4)$ through a patch $P$ corresponding to affine 
parameter range $0<t<t_{0}$, and so are uniquely determined there. Moreover, the orthonormality relations (6.9) are preserved under parallel transport.

Through the identification (7.3), a constant idempotent $\mathbf{U}\left(\mathbf{c}_{\mathrm{r}}\right)$ on $S$ can be written as $\mathbf{U}\left\{\mathbf{W}_{\mathrm{r}}(\mathbf{x})\right\}$; it now takes the form of a bundle of identical idempotents. Any polynomial, in particular $\mathbf{U}\left\{\mathbf{W}_{\mathrm{r}}(\mathbf{x})\right\}$, is parallel transported by (6.12); and since (6.9) are preserved, $\mathbf{U}\left\{\mathbf{W}_{\mathrm{r}}(\mathbf{x})\right\}$ remain idempotent through $P$. The manifold curvature will generally ensure that $W_{r}(x)$ are $x$-dependent for values outside the asymptotically flat region. The S-matrix analogy is the distortion a plane wave as it passes through a region of interaction. However, the structure of the idempotent $U\left\{W_{r}(x)\right\}$ as a projection operator is the same as that of the constant idempotent $\mathbf{U}\left(\mathbf{c}_{\mathbf{r}}\right)$, as will be exemplified in Section 8 . So, in a suitable matrix representation, a primitive idempotent $U\left\{W_{r}(x)\right\}$ can fill out the same (one, or possibly two) column(s) at all points, and therefore can be interpreted as a representation of a particular type of fermion.

The rule for geodesic extension of an idempotent $\mathbf{U}\left(\mathbf{c}_{\mathbf{r}}\right)$ from the asymptotically flat surface $S$ to the patch $P$ is the replacement

$$
\mathbf{c}_{\mathbf{r}} \rightarrow \mathbf{W}_{\mathrm{r}}(\mathbf{x}) \text {, }
$$

defining the position-dependent idempotent on the patch $P$. Since a 'constant vector basis' has no unique well-defined meaning where there is curvature, the replacement (7.7) must be made throughout the spinor (7.1), so that it takes the form

$$
\mathbf{\Psi}(\mathbf{x})=\mathbf{E}\left\{\mathbf{x}, \mathbf{W}_{\mathrm{r}}(\mathbf{x}\} \mathbf{U}\left\{\mathbf{W}_{\mathrm{r}}(\mathbf{x})\right\}\right. \text {. }
$$

This is a polynomial in the spin elements $\mathbf{W}_{\mathrm{r}}(x)$, so that its covariant derivative is, as in (6.7),

$$
\left[\boldsymbol{\delta}_{\mu}, \mathbf{T}(\mathbf{x})\right] \equiv\left[\partial_{\mu}+\mathbf{G}_{\mu}(\mathbf{x}), \mathbf{\Psi}(\mathbf{x})\right]
$$

We must therefore take the 'Dirac operator' for an algebraic spinor to be of the two-sided form

with commutator interaction (6.1).

$$
\mathbf{e}^{\mu}\left[\delta_{\mu}, \Psi(x)\right] \text {, }
$$

Using (7.4) and the orthonormality of the basis $\{\mathbf{W r}\}$, the inverse frame field elements $\mathrm{e}^{\mu}$ in (7.10) can be expressed in the form

So (7.10) can be written in the form

$$
\begin{aligned}
\mathbf{e}^{\mu} & =\eta^{\mathrm{rs}} \mathbf{W}_{\mathrm{g}}\left(\mathbf{e}^{\mu} \cdot \mathbf{W}_{\mathrm{r}}\right) \\
& =\eta^{\mathrm{rs}} \mathbf{W}_{\mathrm{s}} \mathbf{W}_{\mathrm{r}}^{\mu} .
\end{aligned}
$$

$$
\mathbf{W}^{\mathrm{r}} \mathbf{W}_{\mathbf{r}}^{\mu}\left[\boldsymbol{\delta}_{\mu}, \Psi(\mathbf{x})\right] \text {, }
$$

where $W^{r}=\eta^{r s} W_{\mathrm{s}}$. However, the parallel transport condition (6.12) ensures that the term with $r=0$ in the summation vanishes, so that the 'Dirac operator' acting on the spinor (7.8) reduces to

$$
\sum_{r=1}^{n-1} \mathbf{W}^{\mathbf{r}} \mathbf{W}_{\mathbf{r}}^{\mu}\left[\boldsymbol{\delta}_{\mu}, \mathbf{T}(\mathbf{x})\right]
$$

\section{Gravitating Algebraic Spinors: an Example}

In an early paper [1], we proposed a model, based on the Clifford algebra $\mathrm{Cl}_{1,6}$, of the electroweak interactions [13] of leptons, and this model was first generalised to include gravitational interactions [3], and then incorporated into a model describing all of the interactions of a single family of elementary particles [4]. In this model, the constant orthonormal set of basis vectors $\left\{\mathbf{c}_{\mathbf{r}} ; \mathrm{r}\right.$ $=0,1,2, \ldots, 6\}$ of $\mathrm{Cl}_{1,6}$ satisfy

$$
\mathbf{c}_{0}^{2}=-\mathbf{c}_{\mathrm{r}}^{2}=1, \quad(\mathrm{r} \neq 0)
$$

and $\left\{\mathbf{c}_{\mathbf{r}} ; \mathbf{r}=0,1,2,3\right\}$ are taken to be the space-time basis vectors. 
The pseudoscalar $i$ of the algebra commutes with every element, and satisfies $i^{2}=-1$, and so is identified with the unit imaginary. If the complete covariant derivative for the theory is $\partial_{\mu}-\mathbf{\Omega}_{\mu}$, where $\boldsymbol{\Omega}_{\mu}$ is any coordinate vector function on the algebra, then the equation of motion for $\mathbf{i}$ is $\left[\partial_{\mu}\right.$ $\left.\mathbf{\Omega}_{\mu}, \mathbf{i}\right]=0$; and since $\left[\mathbf{\Omega}_{\mu}, \mathbf{i}\right]=0$ for any interaction, it follows that $\mathbf{i}$ is a universal constant. The same argument ensures the consistency of the assumption that 1 , in the fundamental equation (1.1), is common to all points on the manifold.

In this model, the space-time chirality operators are given by

$$
h_{ \pm}=1 / 2(1 \pm i \omega) \text {, }
$$

where $\omega=c_{0123}$ is the space-time pseudoscalar, and the isospin triplet of operators is

$$
\left(\boldsymbol{\rho}_{1}, \boldsymbol{\rho}_{2}, \boldsymbol{\rho}_{3}\right)=\left(\mathbf{c}_{56}, \mathbf{c}_{46}, \mathbf{c}_{45}\right)
$$

Algebraic spinors in flat 7-space are functional elements of the algebra of the form (7.1),

$$
\mathbf{\Psi}(\mathbf{x})=\mathbf{E}\left(\mathbf{x}, \mathbf{c}_{\mathbf{r}}\right) \mathbf{U}\left(\mathbf{c}_{\mathbf{r}}\right)
$$

where $\mathbf{E}\left(x, c_{r}\right)$ is a general element of the algebra and $\mathbf{U}\left(\mathbf{c}_{r}\right)$ is a constant primitive idempotent. It is assumed that $\psi(x)$ depends only on space-time coordinates $\left\{x^{\mu} ; \mu=0,1,2,3\right\}$. In the algebra $\mathrm{Cl}_{1,6}$, primitive idempotents are formed from the maximum number, three, of mutually commuting elements which square to 1 . For reasons which will be explained, we have chosen the triple to be $\left(\mathbf{c}_{03}, \mathbf{i} \mathbf{c}_{12}, \mathbf{i} \mathbf{c}_{45}\right)$, and the primitive idempotent to be

$$
\mathbf{U}=1 / 8\left(1+\mathbf{c}_{03}\right)\left(1-i c_{12}\right)\left(1-i c_{45}\right) \text {. }
$$

To include gravitation, we have assumed $[3,4]$ that the space-time dimensions are curved, but the 'higher dimensions' remain flat. The spinor is assumed to depend only on the space-time coordinates, so that coordinate covariance applies only to $x^{\mu}(\mu=0,1,2,3)$. The spin connection $\mathbf{G}_{\mu}$ contains only the space-time bivectors, and so commutes with $c_{r}(r=4,5,6)$. So $(6.12)$ ensures that the geodesic extensions $W_{r}=c_{r}(r=4,5,6)$ are constant on the patch $P$, so that $\omega$ and the elements (8.2) and (8.3) are also constant.

If we now assume that the spinor is of the form (7.1) on a flat $n-1$ dimensional surface $S$, we can use (7.7) to form the geodesic extension (7.8),

to a patch $P$, with

$$
\mathbf{\Psi}(\mathbf{x})=\mathbf{E}\left\{\mathbf{x}, \mathbf{W}_{\mathbf{r}}(\mathbf{x})\right\} \mathbf{U}\left\{\mathbf{W}_{\mathrm{r}}(\mathbf{x})\right\}
$$

$$
U\left\{W_{r}(x)\right\}=1 / 8\left[1+W_{03}(x)\right]\left[1-i W_{12}(x)\right]\left[1-i W_{45}\right],
$$

where $\mathbf{W}_{\mathrm{rs}}(\mathrm{x})=\mathbf{W}_{\mathrm{r}}(\mathrm{x}) \mathbf{W}_{\mathrm{s}}(\mathrm{x})$, and $\mathbf{W}_{45}=\mathbf{c}_{45}$ is constant on $P$.

The theorems of the previous sections ensure that $W_{r}(x)$ form an orthonormal set on $P$, so that (8.5) defines an idempotent for all $x$. Also, $\mathbf{\Psi}(\mathbf{x})$ has covariant derivative

In (8.6), the commutator

$$
\left[\partial_{\mu}+\mathbf{G}_{\mu}(\mathbf{x}), \boldsymbol{\Psi}(\mathbf{x})\right] \text {. }
$$

$$
\left[\mathbf{G}_{\mu}(\mathbf{x}), \mathbf{\Psi}(\mathbf{x})\right]
$$

is assumed to represent the effect of gravitation on the algebraic spinor $\Psi(x)$. So (7.8) and (8.5) define the form of gravitating algebraic spinors $\Psi(x)$, with two-sided spin connection, in terms of the orthonormal set $\left\{\mathbf{W}_{\mathrm{r}}(\mathbf{x})\right\}$; these are in turn defined as the unique geodesic extensions to the patch $P$ of the constant set $\left\{c_{r}\right\}$ given on the flat surface $S$.

\section{Asymmetry of 'Right-handed' Interactions}

In our earlier papers, the lepton column spinor $\psi(x)$ was assumed to be invariant under gauge transformations operating on the left only, as is assumed in standard gauge theories. The generator of the gauge transformations was of the form

$$
1 / 2 \mathrm{gh}+\boldsymbol{\rho}_{\mathrm{i}} \theta^{\mathrm{i}}(\mathbf{x})+1 / 2 \hat{\mathrm{g}}\left(\mathbf{h}_{-p_{3}}+1\right) \theta^{4}(\mathbf{x})+\mathbf{c}_{\mathrm{pq}} \Theta^{\mathrm{pq}}(\mathbf{x}),
$$


with summations over $i=1,2,3$ and $(p<q)=0,1,2,3$. Then the interaction terms acting on $\psi(x)$, generated by gauge transformations, are

$$
\left[1 / 2 g h_{+} p_{i} V_{\mu}^{i}(x)+1 / 2 \hat{g}\left(h_{-} p_{3}+1\right) V^{4}{ }_{\mu}(x)+W_{p q} G^{p q}{ }_{\mu}(x)\right] \psi(x) \text {, }
$$

with the same operator structure as $(9.1)$, as usual, and with the bivector spin connection (3.12) written as

$$
\mathbf{G}_{\mu}(\mathbf{x})=\mathbf{W}_{\mathrm{pq}}(\mathbf{x}) \mathrm{G}^{\mathrm{pq}}(\mathbf{x}) \text {. }
$$

The terms in (9.2) all contribute to the Lagrangian density. Here, the bivectors $c_{p q}(p, q=0,1,2,3)$ have been replaced by $\mathbf{W}_{\mathrm{pq}}(\mathrm{x})$, in accord with the rule (7.7). The algebraic spinor (7.8) has also been modified by (7.7), so it is necessary to consider the effect of this modification on the variational principle. In flat space theories, we have treated the elements of the 'constant' Clifford algebra as fixed under variations defining the field equations. Since the algebras with bases $\left\{\mathbf{W}_{r}(x)\right\}$ are geodesic extensions of a constant algebra, we assume that they also are fixed under variations defining the field equations. So, under these variations,

(a) only $G^{p q}{ }_{\mu}(x)$ is varied in the bivector term (9.3),

(b) the idempotents, for example (8.5), are not varied, implying that their physical interpretation as fermions is unchanged.

In our more recent paper [12], we considered two-sided gauge transformations in flat spaces, on algebraic spinors of the form given by (7.8) and (8.5). These two-sided gauge transformations give rise to two-sided interaction terms of the form (6.2). For the class of Clifford algebra we consider, we proved that the extra 'right-handed' interaction term, of the general form $-\mathbf{\Psi} \mathbf{A}_{\mu}$, always reduced in the Lagrangian to a multiple of $\Psi(x) 1$, which can be written as $1 \Psi(x)$. Although our idempotents change form under the two-sided gauge transformations, they remain idempotent, and 'expectation values', given by traces, remain invariant.

Now, in our model of the lepton interactions [12], part of the operator multiplying $V_{\mu}^{4}(x)$ in (9.2) is a multiple of 1 , with the same coefficient $\hat{g}$ as the other part of the operator. By making the specific choice (8.5) of primitive idempotent, we were able to simplify the left-hand interaction term (9.2) to

$$
\left[1 / 2 g h_{+} p_{i} V_{\mu}^{i}(x)+1 / 2 \hat{g h} \mathbf{p}_{3} V_{\mu}^{4}(x)+W_{p q}(x) G^{p q}{ }_{\mu}(x)\right] \Psi(x),
$$

This is because the only non-zero contribution to the right hand electroweak interaction term arises from the term

which, using (8.2) and (8.3), gives precisely the term

$$
\Psi(x)^{1 / 2} \hat{g h h}_{3} \mathrm{~V}_{\mu}^{4}(\mathrm{x})
$$

$$
1 / 2 \hat{\mathrm{g}} 1 \mathrm{~V}_{\mu}^{4}(\mathrm{x}) \Psi(\mathrm{x}) \text {. }
$$

Then (9.4) and (9.5) sum to give the required Lagrangian interaction term (9.2).

This means that the generator (9.1) of the gauge transformations can be replaced by the simpler operator

$$
1 / 2 g h_{+} p_{i} \theta^{i}(x)+1 / 2 g h_{-} p_{3} \theta^{4}(x)+W_{p q}(x) \Theta^{p q}(x)
$$

with the electroweak interactions generated only by elements of the isospin algebra. The peculiar term in (9.1) containing 1 no longer appears as a generator.

The electroweak gauge group is now more symmetrical between the two helicities; this is because the choice (8.4) of idempotent to define the ideal representing physically observable spinors is unsymmetrical, and so gives rise to the symmetry-breaking 'right-hand' interaction term (9.5). A different choice of 'physical' idempotent would result in a different symmetry-breaking contribution to (9.2). For example, changing the last bracket in (8.5) to $\left[1+i W_{45}\right]$ would change the algebraic coefficient of $W_{\mu}^{4}(x)$ in $(9.2)$ to (h-p $p_{3}-1$ ). So by choosing (8.5) as the 'physical' 
idempotent, out of the symmetrical set of eight primitive idempotents, we allow the possible introduction of physical asymmetries through the 'right-hand' interaction terms. We state this as a general principle for two-sided interactions:

If, out of a complete symmetrical set of primitive idempotents, one is chosen to define the ideal representing physical states of a fermion, this necessarily introduces an asymmetry in the spinor structure, and may result in the introduction of asymmetrical 'right-hand' physical interactions.

The expression (9.5) is the only term arising from the electroweak terms in (9.4), but the choice (8.5) of idempotent also results in extra 'gravitational' terms arising from the right-hand interaction terms

$$
-\Psi(x) W_{p q} G_{\mu}^{p q}(x) \text {. }
$$

This is because the chosen idempotent depends upon the bivectors $\mathbf{W}_{03}$ and $\mathbf{W}_{12}$, which result in Lagrangian terms proportional to

$$
\left\{1 \mathrm{G}^{03}{ }_{\mu}(\mathrm{x})-\mathrm{i} \mathrm{G}^{12}{ }_{\mu}(\mathrm{x})\right\} \mathbf{\Psi}(\mathrm{x}) \text {. }
$$

Additional gravitational terms of the type (9.7) are hard to avoid in general in this type of theory, since most choices of a maximal set of commuting elements of a Clifford algebra will contain some space-time bivectors. In the present model, the bivector $W_{45}$ in $(8.5)$ has been introduced to produce the correct electroweak interaction. Then the introduction of space-time bivectors in the primitive idempotent is unavoidable. Thus, in this model, the extra gravitational interaction terms (9.7) are a necessary consequence of the form of electroweak interactions. The unusual nature of these terms will be discussed in the next section.

Our 'two-sided' interactions differ from those of Trayling and Baylis [33], in a model also based on a seven-dimensional Clifford algebra. In their model, they use a complete set of idempotents to incorporate all particles and antiparticles of one family, and different gauge groups act on the left and the right This allows them to introduce all non-gravitational interactions within their sevendimensional model, whereas we have used eleven dimensions [4,6]. So Trayling and Baylis' interaction terms are only 'one-sided', compared with our two-sided interactions. They do not introduce gravitation, and another difference between their work and ours is that they introduce a Higgs field, while we generate the boson mass matrix by interpreting the fermion mass terms as an interaction with the frame field $[1,3,4]$. We shall discuss this interaction term in Section 10.

\section{Discussion}

Many of the properties of Clifford manifolds are shared with other definitions of metric manifolds. Our reasons for singling out this structure, based on equations (1.1) and (1.2), are, first, that it is a very specific, simple structure; and second, that we have gone some way, within this simple structure, to model the properties of particles of a family of elementary particles and all of their interactions, including gravitation. In the first two sections of this paper, the properties of coordinate groups on a manifold, and of the spin group on tangent spaces, have been developed. Various fields have been classified by their transformation properties under these groups.

The unique connection properties of different fields have then been derived from postulates of covariance under the two groups, together with the 'uniformity assumption' of grade structure on the manifold. It has been known for many years that the frame field, fundamental to Clifford manifolds, requires both vector and spin connections. A basic result of this paper is that, on a Clifford manifold, the frame field is necessarily covariantly constant. This result depends in an 
essential way on the conciseness of the Clifford manifold concept, which incorporates both Riemannian curvature and spin structure in the basic formula (1.1), together with particle gauge generators in higher dimensional models. This conciseness can be contrasted, for example, with Weinberg's approach [14], in which a tetrad of Riemannian fields and a spin group are introduced separately. In another approach, Brill and Cohen [34] treat the tetrad frame and the Dirac matrices as distinct entities, obeying the rules of Grassmann and Clifford algebras respectively; they then have to assume that the Dirac matrices are the same in every reference system. Ruth Farwell and I have long taken the view, in sympathy with Fock and Ivanenko [19], that the frame field is both a geometrical and a physical object. We also believe that a single Clifford algebraic structure, based on (1.1), should provide a unified view of geometry and physics.

The covariant constancy of the frame field, with both vector and spin connections, leads to one of the most important results of this paper, a fundamental linking of a Riemannian vector field with a 'spin element', which has 'two-sided' covariant derivative of the form (6.5). This natural and inevitable linkage is quite different from the assumptions of supersymmetry, and does not involve the introduction of unobserved supersymmetric partners. As a consequence of this linkage, a set of Riemannian fields, orthonormal at a non-degenerate point on a manifold, is related to a set of spin elements orthonormal in the associated tangent space; orthonormal sets of spin elements at different points can be regarded as a bundle of position-dependent vector bases of a Clifford algebra. So position-dependent idempotents can then be defined in neighbourhoods of a manifold, which possess a two-sided spin covariant derivative. It is therefore possible to relate, by parallel transport, the algebras and idempotents at points along a path on the manifold, using this spin covariant derivative. This contrasts with the assumption of Brill and Cohen [34] of a constant representation of the Dirac matrices, which has no covariant meaning.

Although it is possible to use the two-sided spin connection to define values for idempotents, and hence spinors, over suitable curved patches of an $n$-dimensional manifold $M$, there is generally no unique way of doing this. There is thus an ambiguity in defining a curved space generalization of constant idempotents, which are used to define algebraic spinors in flat spaces. We are proposing a method of giving a unique definition of idempotents on a patch of $\mathrm{M}$, by geodesic extension of a constant idempotent from an $n-1$ dimensional flat surface lying in a flat region of $M$. This process is analogous to the distortion, in a 'region of interaction', of an asymptotically plane wave in scattering theory. This analogy suggests that it would also be useful to give a unique definition of idempotents and spinors which are asymptotically represented by 'outgoing' spherical harmonics. The well-established methods of Clifford analysis might well be used to develop this idea, which is beyond the scope of this paper.

The two-sided 'Dirac equation' based on (7.10) or (7.12) is proposed as the equation of motion of an algebraic spinor subject to gravitation. Through (6.4), this equation is related to a set of quasilinear equations governing a set of Riemannian fields satisfying the orthonormality condition (5.7). But the most significant effect of the new 'right-hand' interaction term is the inevitable appearance, in the model of Sections 8 and 9, of the asymmetric interaction terms (9.7). They have a very interesting form, since their operator dependence, on $\mathbf{I}$, is that required for a 'cosmological constant'. Also, their magnitude will be of the same order as the standard gravitational terms, which is also desirable. However, these terms are not constant, and they are spacially unsymmetric. It is natural to ask whether they could act macroscopically as a cosmological term affecting the rate of expansion of the universe. It is also natural to note that the chosen idempotent (8.5) is time asymmetric through the factor $\left(1+W_{03}\right)$, resulting in the interaction term $1 \mathrm{G}^{03}{ }_{\mu}(x)$ in $(9.7)$, and to ask whether this term can be linked to the observed time asymmetry of the universe. These new 
'right-hand' interaction terms appear at single-particle level, whereas time asymmetry and a cosmological 'constant' are macroscopic concepts. It is not within the scope of this paper to work out the macroscopic or cosmological effects of these terms.

The frame field, introduced in (1.1), is fundamental to the concept of Clifford manifolds. Using the absolute identity

$$
\gamma^{\mu} \gamma_{\mu}=41 \text {, }
$$

we have expressed the mass term in the Dirac equation as an interaction with the frame field $[1,2,3,4]$. Since the bivectors of the Dirac algebra have been used as generators of gauge transformations in (9.1) and (9.6), it is natural to ask whether the vectors of the algebra cannot be regarded as translational gauge generators, completing the Poincare group. Many authors, including Kibble [11], Hehl et al. [35], and Lasenby et al. [36], have used translational generators in gravitational gauge theories. However, our models include electroweak interactions, requiring the specific introduction of the chirality operators $h_{ \pm}$into the Lagrangian, as in (9.1). These operators do not commute with the vectors of the algebra, and it is this non-commutation which has enabled us to explain the boson mass ratios without introducing Higgs fields $[1,3,4]$. The chirality operators are introduced because we use 4-component neutrinos; this allows us to introduce neutrino masses, which now seem to be demanded by experiment. If we included the Clifford vectors as gauge generators, the full gauge group would be enlarged, requiring the introduction of boson fields which are not observed. So, in our particular models, the vielbein field $h_{\mu}{ }^{\mathrm{T}}(\mathrm{x})$ is not a gauge field, but simply the coefficients in the expansion (2.3) of the frame field in terms of an orthonormal vector basis. Since we regard the frame field as the fundamental field defining a Clifford manifold, it is not inconsistent to ascribe different properties to it and to the gauge-generated fields which describe particle interactions.

Hehl et al. [35], like Kibble [11], use the full Poincare group as an infinitesmal gauge group, but their analysis leads to the introduction of torsion. In contrast, torsion is built in to a Clifford manifold through the spin connection, as we discussed in Section 4. They use a Lagrangian purely quadratic in the curvature, which Bicknell [37] has noted presents difficulty in the coupling of gravitation to matter. Our models $[2,3,4]$ give a 'free' gravitational Lagragian containing both linear and quadratic curvature terms, and we have pointed out [2] that inflation in the early universe can be based on a suitable combination of the two types of term. Hehl et al. interpret a part of their interactions generated by the Poincare group as strong interactions, but they do not meet up with our difficulty arising from the helicity asymmetric electroweak interactions.

The theory of Lasenby et al. [36] is based on Clifford algebra, expressed in Hestenes-Sobczyk notation [22]. Their translational gauge transformations are finite, and the interpretation of their theory is in terms of a flat space-time. The physical predictions overlap strongly, but not completely, with Poincare gravitational gauge theories. In this formulation, spinors can be operated on 'on both sides', but they do not introduce two-sided interactions of the form (6.2) and (8.7). Their extensive applications include electromagnetic phenomena, but these are helicity symmetric, so that here is no possibility of non-commutation of the relevant generators with translational gauge generators. The authors who do include helicity asymmetric interactions in their Clifford algebraic models are Trayling and Baylis [33]; but they do not deal with gravitation.

We have remarked in earlier papers on the difference between our treatment of the space-time dimensions and the higher dimensions. This discrepancy is even more obvious in the present paper. In the model of Sections 8 and 9 , the gauge transformations are generated within both the spacetime dimensions $0,1,2,3$ and the isospin dimensions 4,5,6. However, the covariant derivatives, and hence the dynamics and the curvature, are restricted to space-time. The model cries out for the 
extension of dynamics and curvature to the higher dimensions. This would allow the compaction of higher dimensions, which is not a novel idea, with the introduction of the inverse Planck length as a curvature of the higher dimensions, and might perhaps offer the chance of explaining the fermion mass spectrum in terms of higher-dimensional dynamics. Since these higher dimensions are, in our models, linked to specific gauge groups, we have a strong guide to the interpretation of the associated dynamical degrees of freedom. The author intends to investigate this idea in the near future.

\section{Acknowledgments}

The author has benefited from useful comments by Professor MacCallum of QMW, Professor Baylis of the University of Windsor, and Dr Ryder and Dr Shank of the University of Kent. He is also grateful to Carol Chisholm for providing a translation of Schrodinger's paper [24], and to the referees, whose suggestions have led to major improvements to this paper. The paper is a continuation of work carried out over many years in collaboration with Dr Ruth Farwell of South Bank University, and is based on ideas developed during this collaboration.

\section{Appendix A}

The following formula is a generalisation of a familiar formula for the Dirac algebra [16,17]. A general $k$-vector of a Clifford algebra is denoted by $\mathbf{E}_{(\mathbf{k})}$. As usual, upper suffix vectors are defined by $e^{\mu}=g^{\mu v} e_{v}$. The formula we shall establish is

$$
\mathrm{e}^{\mu} \mathbf{E}_{(\mathrm{k})} \mathbf{e}_{\mu}=\mathbf{e}_{\mu} \mathbf{E}_{(\mathrm{k})} \mathbf{e}^{\mu}=(-1)^{\mathrm{k}}(\mathrm{n}-2 \mathrm{k}) \mathbf{E}_{(\mathrm{k})}
$$

To prove this result, note that the expansion (2.3)

$$
\mathbf{e}_{\mu}(x)=h_{\mu}{ }^{\prime}(x) c_{r}(x)
$$

in terms of a local orthonormal basis $\left\{c_{r}(x)\right\}$ implies that

$$
e^{p}(x)=k_{r}^{\mu}(x) c^{x}(x)
$$

where the matrix $\left(k_{r}{ }^{\mu}\right)$ is the inverse of $\left(h_{\mu}{ }^{T}\right)$, and $c^{r}(x)=\eta^{r s} c_{s}(x)$, so that, for each value of $r$,

$$
c^{r} \mathbf{c}_{\mathbf{r}}=1 \text {. }
$$

Then if $\mathbf{E}(\mathbf{x})$ is any element of the algebra,

$$
\mathbf{e}^{\mu} \mathbf{E e}_{\mu}=\mathbf{e}_{\mu} \mathbf{E e}^{\mu}=\mathbf{c}^{\mathrm{r}} \mathbf{E} c_{\mathrm{r}}
$$

To establish (A1), note that, for $k$ even, any basis element of grade $k$ anticommutes the $k$ basis vectors comprising that element, and commutes with the $n-k$ other basis vectors. Since $\mathbf{E}_{(k)}$ is the sum of such basis elements, the sum $\mathbf{c}^{\mathrm{r}} \mathbf{E}_{(\mathrm{k})} \mathbf{c}_{\mathrm{r}}=(\mathrm{n}-\mathrm{k}-\mathrm{k}) \mathbf{E}_{(\mathrm{k})}$. Using (A5), (A1) is established for even k. A similar argument establishes (A1) for odd $\mathrm{k}$.

\section{Appendix B}

To establish Theorem 1, consider the double commutator given by substituting $\mathbf{G}_{\mu}$ from (3.12) into the commutator in (4.6). This gives

where

$$
\left[\left[\mathbf{e}^{\rho},\left(D_{\mu} \mathbf{e}_{p}\right)\right], \mathbf{e}_{v}\right]=\mathbf{T}_{1}+\mathbf{T}_{2},
$$

$$
T_{1}=-\Gamma_{\mu \rho}^{\lambda}\left(e^{\rho} e_{\lambda} e_{\nu}-e_{\lambda} e^{\rho} e_{v}-e_{\nu} e^{\rho} e_{\lambda}+e_{\nu} e_{\lambda} e^{p}\right)
$$

and

$$
\mathbf{T}_{2}=\mathbf{e}^{\rho}\left(\partial_{\mu} \mathbf{e}_{\rho}\right) \mathbf{e}_{v}-\left(\partial_{\mu} \mathbf{e}_{\rho}\right) \mathbf{e}^{\rho} \mathbf{e}_{v}-\mathbf{e}_{v} \mathbf{e}^{\rho}\left(\partial_{\mu} \mathbf{e}_{\rho}\right)+\mathbf{e}_{v}\left(\partial_{\mu} \mathbf{e}_{\rho}\right) \mathbf{e}^{\rho}
$$


Using the formula

$$
\left\{\mathbf{e}^{p}, \mathbf{e}_{v}\right\}=2 \mathbf{I} \delta^{p}{ }_{v} .
$$

derived from (1.1), and (1.1) itself, (B2) reduces to

$$
\begin{aligned}
\mathbf{T}_{1} & =4 \Gamma_{\mu \rho}^{\lambda}\left(\delta^{\rho}{ }_{v} \mathbf{e}_{\lambda}-g_{\lambda \nu} \mathbf{e}^{\rho}\right) \\
& =4 \Gamma_{\mu v} \mathbf{e}_{\lambda}-4 \Gamma_{\mu \rho v} \mathbf{e}^{\rho} .
\end{aligned}
$$

To simplify the sum of the first and third terms in (B3), add and subtract $\mathrm{e}^{\rho} \mathrm{e}_{\nu}\left(\partial_{\mu} \mathrm{e}_{\mathrm{\rho}}\right)$. This gives

$$
\mathbf{e}^{\rho}\left[2 \partial_{\mu} g_{\rho v}-e_{p}\left(\partial_{\mu} e_{v}\right)-\left(\partial_{\mu} e_{v}\right) e_{\rho}\right]-2 \delta_{v}^{p_{v}}\left(\partial_{\mu} e_{\rho}\right)
$$

Since $\left(\partial_{\mu} \mathbf{e}_{v}\right)$ is a spin vector, formula (A1) with $n=0$ and $n=1$ reduces (B6) to

$$
2\left(\partial_{\mu} \mathrm{g}_{\rho v}\right) \mathbf{e}^{\rho}-4 \partial_{\mu} \mathbf{e}_{v}
$$

It is interesting that the coefficients $n$ from (A1) cancel out, so that (B7) is true for manifolds of any dimensionality and signature.

In a very similar way, the sum of the second and fourth terms in (B3) reduce to the same expression (B7). Thus (B3) is equal to

$$
4\left(\partial_{\mu} g_{p v}\right) e^{\rho}-8 \partial_{\mu} \mathbf{e}_{v}
$$

The second term in (B5) can now be combined with the first term in (B7) to give

$$
4\left(\partial_{\mu} g_{\rho v}\right) \mathbf{e}^{\rho}-4 \Gamma_{\mu \rho v} e^{\rho}=4 \Gamma_{\mu \nu \rho} e^{\rho} .
$$

So, adding (B5) and (B7), (B1) becomes

$$
\begin{gathered}
{\left[\left[\mathbf{e}^{\rho},\left(D_{\mu} \mathbf{e}_{\rho}\right)\right], \mathbf{e}_{v}\right]=\mathbf{T}_{1}+\mathbf{T}_{2}} \\
=8 \Gamma_{\mu v \rho} \mathbf{e}^{\rho}-8 \partial_{\mu} \mathbf{e}_{v} .
\end{gathered}
$$

This establishes the Covariant Constancy İdentity (4.6).

\section{References}

1. J.S.R.Chisholm and R.S.Farwell 1987, Electroweak Spin Gauge Theories and the Frame Field, J.Phys.A20, 6561

2. J.S.R.Chisholm and R.S.Farwell 1988, Gravity and the Frame Field, Gen. Rel, and Gravitation, 20, 371

3. J.S.R.Chisholm and R.S.Farwell 1988, Unified Spin Gauge Theory of Electroweak and Gravitational Interactions, J.Phys.A22, 1059

4. J.S.R.Chisholm and R.S.Farwell 1990, Unified Spin Gauge Theories of the Four Fundamental Forces, 'The Interface of Mathematics and Physics', eds. D.G.Quillan et al, 193 (OUP)

5. J.S.R.Chisholm and R.S.Farwell 1992, A Fermion-boson Mass Relation and the Top Mass, J.Phys.G18, L117

6. J.S.R.Chisholm and R.S.Farwell 1993, Spin Gauge Theories: Principles and Predictions, 'Clifford Algebras and their Applications in Mathematical Physics', eds. F.Brackx et al. (Kluwer)

7. J.S.R.Chisholm and R.S.Farwell 1991, Clifford Approach to Metric Manifolds, Supp. ai Rendiconti del Circ. Mat. di Palermo 2, 26, 123

8. I.R.Porteous 1995, 'Clifford Algebras and the Classical Groups', Chapter 16 (CUP)

9. S.Weinberg 1972, 'Gravitation and Cosmology' (John Wiley and Sons)

10. R.Utiyama 1955, Invariant Theoretical Interpretation of Interaction, Phys. Rev. 101, 5, 1597

11. T.W.B.Kibble 1961, Lorentz Invariance and the Gravitational Field, J.Math.Phys. 2, 2, 212

12. J.S.R.Chisholm and R.S.Farwell 1999, Gauge Transformations of Spinors within a Clifford Algebraic Structure, J.Phys. A32, 2805

13. S.Glashow 1961, Partial-symmetries of Weak Interactions, Nucl. Phys. 22, 579

14. S.Weinberg, loc.cit. $365-373$ 
15. W.Pezzaglia 2000, Dimensionally Democratic Calculus and Principles of Polydimensional Physics, in 'Clifford Algebras and their Applications in Mathematical Physics', vol. 1, 101, eds. R.Ablamowicz and B.Fauser (Birkhauser)

16. E.Caianello and S.Fubini 1952, On the Algorithm of Dirac Spurs, Nuovo Cimento Serie 9, 9, 1218

17. J.S.R.Chisholm 1952, The Calculation of S-matrix Elements and Magnetic Moments, Ph.D thesis (Cambridge)

18. E.E.Fairchild 1977, Yang-Mills Formulation of Gravitational Dynamics, Phys.Rev. D16, 2438

19. V.Fock and D.Ivanenko 1929. Geometrie Quantique Lineare et Deplacement Parallele, Comptes Rendues 188, 1470

20. A.Connes $2000, A$ Short Survey of Non-commutative Geometry, J.Math. Phys. 41, 3832

21. H.S.Green 1958. Spinor Fields in General Relativity, Proc. Roy. Soc.A245, 521

22. D.Hestenes and G.Sobczyk 1984, 'Clifford Algebra to Geometric Calculus', Chapter 6, (D.Reidel)

23. S.Chern 1989, Vector Bundles with a Connection, 'Global Differential Geometry' (Math. Assn. America)

24. E.Schrodinger 1932, Diracsches Elektron im Schwerefeld I, Preuss. Akad. Wiss. (Berlin), Mitt. der Phys-Math, 105

25. D.B.Brill and J.Wheeler 1957, Interaction of Neutrinos and Gravitational Fields, Rev. Mod. Phys. 29,465

26. J.G.Fletcher 1958, Dirac Matrices in Riemannian Space, Il Nuovo Cimento 8, 3, 451

27. J.G.Loos 1963, Spin Connection in General Relativity, Ann. Physics 25, 91

28. R.M.Wald 1984, 'General Relativity', Section 3.3 (University of Chicago)

29. R.L.Bishop and R.J.Crittenden 1964, 'Geometry of Manifolds', Chapter 8 (Academic)

30. S.S.Chem, W.H.Chen and K.S.Lam 1999, 'Lectures on Differential Geometry', Chapter 5 (World Scientific)

31. W.L.Bade and H.Jehle 1953, An Introduction to Spinors, Rev. Mod. Phys. 25, 714

32. R.M.Wald, loc. cit, 283-285

33. G.Trayling and W.E.Baylis 2001, A Geometrical Basis for the Standard Model Gauge Group, J.Phys. A34, 3309

34. D.B.Brill and J.M.Cohen 1966, Cartan Frames and General Relativistic Dirac Equation, J.Math. Phys. 7, 2, 238

35. F.W.Hehl, J.Nitsch and P.van der Heyde 1980, Gravitation and Poincare Gauge Field Theory with Quadratic Lagrangian, 'Einstein Commemorative Volume', 67 (Plenum)

36. A.Lasenby, C.Doran and S.Gull 1998, Gravity, Gauge Theories and Geometric Algebra, Phil. Trans. Roy. Soc. 356, 487

37. G.V.Bicknell 1974, Non-viability of Gravitational Theory based on a Quadratic Lagrangian, J.Phys. A7, 1061 